\title{
SUCCESSIVE APPROXIMATIONS IN ORDERED VECTOR SPACES AND GLOBAL SOLUTIONS OF NONLINEAR VOLTERRA INTEGRAL EQUATIONS
}

\author{
BY
}

TERRENCE S. MCDERMOTT

\begin{abstract}
Conditions are found under which a nonlinear operator in an ordered topological vector space will have a fixed point. This result is applied to study a nonlinear Volterra integral operator in the space of continuous, real valued functions on $[0, \infty)$ equipped with the topology of uniform convergence on compact subsets. Two theorems on the global existence of solutions to the related Volterra integral equation as limits of successive approximations are proved in this manner.
\end{abstract}

Introduction. In the first section of this paper, we establish conditions under which an operator equation $F x=x$ in a partially ordered topological vector space, $X$, will have a solution, unique in a certain class, as a limit of successive approximations. The conditions involve a related operator, $G$, for which certain inequalities hold, guaranteeing $F$ is contractive in an appropriate space.

The second and third sections are given over to two examples in which this general method is applied to establish existence of global solutions for the scalar nonlinear Volterra integral equation

$$
x(t)=y(t)+\int_{0}^{t} k(t, s) f(s, x(s)) d s,
$$

where $X$ will be the space of continuous functions on $[0, \infty)$ under its natural ordering.

In the example of $\S 1$, the related operator, $G$, is effectively linear, while $f$ of $(0.1)$ is required to satisfy a loose local Lipschitz condition similar to the type considered by Miller and Sell [7]. The example to which $\$ 2$ is devoted involves a nonlinear $G$, and the Lipschitz condition is replaced by a condition of the form

$$
\left|f\left(t, x_{1}\right)-f\left(t, x_{2}\right)\right| \leqq \varphi(t) \psi\left(\left|x_{1}-x_{2}\right|\right)
$$

where $\psi$ is continuous, monotone increasing, and $\int_{K}^{\infty}(1 / \psi(x)) d x=+\infty$ for some $K>0$.

The work in this article relates to some earlier work as follows. The underlying method is related in concept to the works of Corduneanu [2], [3], Lakshmikantham

Received by the editors March 1, 1971.

AMS 1970 subject classifications. Primary 47H15, 47H10, 45D05, 45G99.

Key words and phrases. Ordered topological vector space, fixed points, Volterra integral equation, global solution, successive approximations.

Copyright (C) 1972, American Mathematical Society 
and Leela [4, Chapter 5], and the author [6]. The criterion obtained in $\$ 2$ is related to, but in certain respects less restrictive than, that given by A. Strauss [10]. The results of $\S 3$ are, in special cases, related to some results of Nohel [8].

1. A fixed point theorem. In this section, $X$ is a partially ordered, real, Hausdorff linear topological space in which the symmetric order-closed intervals $\left[-x_{0}, x_{0}\right]=\left\{x \in X:-x_{0} \leqq x \leqq x_{0}\right\}$ are bounded and sequentially closed. The real numbers will be denoted by $R$, the null vector of $X$ by $\theta$.

THEOREM 1.1. Let $X$ be sequentially complete and $F: X \rightarrow X$ a sequentially continuous mapping such that, for some $x_{1} \geqq \theta, F\left(\left[-x_{1}, x_{1}\right]\right) \subset\left[-x_{1}, x_{1}\right]$. Suppose $G: X \rightarrow X$ is a mapping satisfying

(a) $G(x) \geqq \theta \forall x \in X$.

(b) $G([-x, x]) \subset[\theta, G(x)]$.

(c) $\exists m, 0<m \leqq 2$, such that $0 \leqq \lambda \leqq m \Rightarrow G(\lambda x) \leqq \lambda G(x)$.

(d) $\forall x, y \in\left[-x_{1}, x_{1}\right],-G(x-y) \leqq F(x)-F(y) \leqq G(x-y)$.

(e) $\exists x_{2} \geqq \theta$ satisfying the inequalities $(1 / \delta) G\left(x_{2}\right) \leqq x_{2},(2 / m) x_{1} \leqq x_{2}$ for some $\delta$, $0<\delta<1$.

Then the equation $x=F(x)$ has one and only one solution in $\left[-x_{1}, x_{1}\right]$.

Proof. First, notice from (e) that $x_{1} \leqq(m / 2) x_{2}$ and that, consequently, $\left[-x_{1}, x_{1}\right]$ $\subset\left[-(m / 2) x_{2},(m / 2) x_{2}\right]$. Let $X_{2}$ be the subspace of $X$ spanned by $\left[-x_{2}, x_{2}\right]$ equipped with the norm (since $\left[-x_{2}, x_{2}\right]$ is bounded, balanced and convex)

$$
\|x\|_{2}=\inf \left\{\lambda>0: x \in\left[-\lambda x_{2}, \lambda x_{2}\right]\right\}, \quad x \in X_{2} \text {. }
$$

If $x, y$ are in $\left[-x_{1}, x_{1}\right]$, then $(x-y) \in\left[-m x_{2}, m x_{2}\right]$. Hence $\|x-y\|_{2} \leqq m$. Let $0<\lambda \leqq m$, then, using (a), (b), (c), and (d), if $(x-y) \in\left[-\lambda x_{2}, \lambda x_{2}\right]$, we have

$-\lambda G\left(x_{2}\right) \leqq-G\left(\lambda x_{2}\right) \leqq-G(x-y) \leqq F(x)-F(y) \leqq G(x-y) \leqq G\left(\lambda x_{2}\right) \leqq \lambda G\left(x_{2}\right)$.

Consequently, since $G\left(x_{2}\right) \leqq \delta x_{2}$,

$$
\|F(x)-F(y)\|_{2} \leqq \delta\|x-y\|_{2} \text { for all } x, y \in\left[-x_{1}, x_{1}\right] .
$$

The usual argument shows that the sequence

$$
x_{0}=F(\theta) \in\left[-x_{1}, x_{1}\right], \quad x_{n}=F\left(x_{n-1}\right), \quad n \geqq 1,
$$

is Cauchy with respect to $\|\cdot\|_{2}$. By hypothesis, the sequence lies in $\left[-x_{1}, x_{1}\right]$. Since [ $\left.-x_{1}, x_{1}\right]$ is bounded in $X, x_{n}$ is therefore Cauchy in the topology of $X$, hence converges by the sequential completeness of $X$ to some point $x_{0}$. Since $F$ is sequentially continuous, and $\left[-x_{1}, x_{1}\right]$ is sequentially closed, we are finished except for uniqueness in $\left[-x_{1}, x_{1}\right]$, which follows by the standard argument.

THEOREM 1.2. Suppose $G: X \rightarrow X$ satisfies conditions (a) and (b) of Theorem 1.1 and $F: X \rightarrow X$ satisfies $-G(x) \leqq F(x)-F(\theta) \leqq G(x)$ for all $x \in X$. Then, if the inequality $x_{3}+G(x) \leqq x$ has a solution $x=x_{1} \geqq \theta$ for some $x_{3} \geqq \theta$ with $-x_{3} \leqq F(\theta) \leqq x_{3}$, $F:\left[-x_{1}, x_{1}\right] \rightarrow\left[-x_{1}, x_{1}\right]$. 
Proof. $-G(x) \leqq F(x)-F(\theta) \leqq G(x)$. Hence, $-G(x)+F(\theta) \leqq F(x) \leqq G(x)+F(\theta)$. If $-x_{1} \leqq x \leqq x_{1}$, then $G(x) \leqq G\left(x_{1}\right)$ by (b). Therefore

$-x_{1} \leqq-G\left(x_{1}\right)-x_{3} \leqq-G\left(x_{1}\right)+F(\theta) \leqq F(x) \leqq G\left(x_{1}\right)+F(\theta) \leqq G\left(x_{1}\right)+x_{3} \leqq x_{1}$.

2. In this section, we shall be concerned with functions that are locally $L^{2}$ or that are locally $L^{2}$ kernels (see Smithies [9]). In the way of notation, the norms of $L^{2}[0, t]$ and $L^{2}([0, t] \times[0, t])$ will be denoted by $\|\cdot\|_{t}$ and $\|\cdot\|_{t \times t}$ respectively. $C\left(R^{+}\right)$will denote the vector space of continuous functions on $[0, \infty)$ with values in $R$ under the topology of uniform convergence on compact subsets, where $R$ denotes the real numbers, and $R^{+}=\{x \in R: x \geqq 0\}$. If $x, y \in C\left(R^{+}\right)$we may write $x \geqq y$ instead of $x(t) \geqq y(t), t \in R^{+}$.

Definition 2.1. A function $g: R^{+} \rightarrow R$ will be called locally $L^{2}$-compatible with $k: R^{+} \times R^{+} \rightarrow R$ if $g$ is measurable and

(2.1) $\forall T>0, k(t, s) g(s)$ is an $L^{2}$ kernel on $[0, T] \times[0, T]$.

(2.2) $\forall T>0, \lim _{t \rightarrow T} \int_{0}^{T}[(k(t, s)-k(T, s)) g(s)]^{2} d s=0$.

LEMMA 2.1. If $g$ is locally $L^{2}$-compatible with $k$, then

$$
\sup _{t \leqq T}\|k(t, \cdot) g(\cdot)\|_{t}<\infty .
$$

Proof. The proof follows by observing that (2.2) implies that the map $t \mapsto k(t, \cdot) g(\cdot)$ from $[0, T]$ into $L^{2}[0, T]$ is continuous, while $[0, T]$ is compact and $\|k(t, \cdot) g(\cdot)\|_{t} \leqq\|k(t, \cdot) g(\cdot)\|_{T}$ if $t \leqq T$.

THEOREM 2.1. Let $g: R^{+} \rightarrow R$ be locally $L^{2}$-compatible with $k$ and suppose $y \in C\left(R^{+}\right)$is nonnegative. Then, the linear integral equation

$$
x(t)-y(t)=\int_{0}^{t}|k(t, s) g(s)| x(s) d s
$$

has a unique nonnegative solution in $C\left(R^{+}\right)$.

Proof. Let $H$ denote the integral operator defined by the right-hand side of (2.4). It suffices to show that $x-y=H x$ has a unique continuous solution on $[0, T]$ for arbitrary $T>0$. The $L^{2}$ theory for linear Volterra integral equations guarantees on $[0, T]$ (see Smithies [9]) that the unique solution of $(2.4)$ in $L^{2}[0, T]$ can be written

$$
x=y+\sum_{n=1}^{\infty} H^{n} y,
$$

where $H^{n}$ is the $n$th iterate of $H$, and that the kernel $K_{n}$ of $H^{n}$ satisfies

$$
\left|K_{n}(s, t)\right| \leqq\left(\|k(t, s) g(s)\|_{T \times T}^{n-1} /((n-1) !)^{1 / 2}\right) k_{1}(t) k_{2}(s),
$$

where

$$
k_{1}(t)=\left[\int_{0}^{t}|k(t, s) g(s)|^{2} d s\right]^{1 / 2}=\|k(t, \cdot) g(\cdot)\|_{t}
$$


and

$$
k_{2}(s)=\left[\int_{s}^{T}|k(t, s) g(s)|^{2} d t\right]^{1 / 2}
$$

The solution $x$ is continuous, since $H: C\left(R^{+}\right) \rightarrow C\left(R^{+}\right)$and the convergence of (2.5) is uniform on each $[0, T]$. The first assertion follows from the fact that if $x \in C\left(R^{+}\right)$and $t_{0} \in R^{+}$then

$$
\left|H x(t)-H x\left(t_{0}\right)\right| \leqq\left\|k(t, \cdot) g(\cdot)-k\left(t_{0}, \cdot\right) g(\cdot)\right\|_{t_{0}}^{1 / 2}\|x\|_{t_{0}}^{1 / 2}+\left|\int_{t_{0}}^{t}\right| k(t, s) g(s)|x(s) d s| .
$$

The first term will be small by (2.2) if $\left|t-t_{0}\right|$ is sufficiently small. If $t<t_{0}+\varepsilon=t_{1}$, $\varepsilon>0$, then

$$
\begin{aligned}
\left|\int_{t_{0}}^{t}\right| k(t, s) g(s)|x(s) d s| & \leqq\left[\int_{t_{0}}^{t}|k(t, s) g(s)|^{2} d s\right]^{1 / 2}\left[\int_{t_{0}}^{t}|x(s)|^{2} d s\right]^{1 / 2} \\
& \leqq\|k(t, \cdot) g(\cdot)\|_{t_{1}}\left[\int_{t_{0}}^{t}|x(s)|^{2} d s\right]^{1 / 2}
\end{aligned}
$$

which tends to 0 as $t \rightarrow t_{0}$. Thus $H: C\left(R^{+}\right) \rightarrow C\left(R^{+}\right)$. That the convergence of (2.4) is uniform on $[0, T]$ follows by the Weierstrass $M$-test, since for all $t \leqq T$,

$$
\left|H^{n} y(t)\right| \leqq \int_{0}^{t}\left|K_{n}(s, t)\right||y(s)| d s \leqq \frac{\left\|K_{1}\right\|_{T \times T}^{n-1}}{((n-1) !)^{1 / 2}}\left|k_{1}(t)\right| \int_{0}^{T}\left|k_{2}(s)\right||y(s)| d s,
$$

and, by Lemma $2.1, \exists M>0$ such that $\left|k_{1}(t)\right| \leqq M$ for $t \leqq T$. Hence, using the Cauchy-Schwartz inequality,

$$
\left|H^{n} y(t)\right| \leqq M\left\|K_{n}\right\|_{T \times T}^{n+1}\|y\|_{T}^{2} /((n-1) !)^{1 / 2} .
$$

THEOREM 2.2. Let $f: R^{+} \times R \rightarrow R$ be measurable and suppose there exists a function, $\varphi$, locally $L^{2}$-compatible with $k: R^{+} \times R^{+} \rightarrow R$ such that

$$
|f(t, x)| \leqq \varphi(t)|x| \text {. }
$$

Then, the operator $F$ defined on $C\left(R^{+}\right)$by

$$
F x(t)=\int_{0}^{t} k(t, s) f(s, x(s)) d s+y(t), \quad x \in C\left(R^{+}\right),
$$

takes its values $F x$ in $C\left(R^{+}\right)$.

Proof. That $F x$ is defined follows from the measurability of $f$ and $k$ and the integrability of $k(t, s) \varphi(s)|x(s)|$ for each $t$. The proof that $F x$ is in $C\left(R^{+}\right)$is similar to that for $H x$ in Theorem 2.1 .

THEOREM 2.3. Let the hypotheses and notation of Theorem 2.2 hold. If, in addition, for each pair $S>0, T>0$ there exists a function $m_{S T}: R^{+} \rightarrow R$ that is locally $L^{2}-$ compatible with $k$ such that

$$
\left|f\left(t, x_{1}\right)-f\left(t, x_{2}\right)\right| \leqq m_{S T}(t)\left|x_{1}-x_{2}\right|
$$

for all $t \in[0, T], x_{1}, x_{2} \in[-S, S]$, then $F: C\left(R^{+}\right) \rightarrow C\left(R^{+}\right)$is continuous. 
Proof. Let $x_{0} \in C\left(R^{+}\right)$. For any $x \in C\left(R^{+}\right)$,

$$
\begin{aligned}
\left|F x(t)-F x_{0}(t)\right| & =\left|\int_{0}^{t} k(t, s)\left[f(s, x(s))-f\left(s, x_{0}(s)\right)\right] d s\right| \\
& \leqq \int_{0}^{t}\left|k(t, s) m_{S T}(s)\right|\left|x(s)-x_{0}(s)\right| d s \\
& \leqq\left[\int_{0}^{t}\left|k(t, s) m_{S T}(s)\right|^{2} d s\right]^{1 / 2}\left[\int_{0}^{t}\left|x(s)-x_{0}(s)\right|^{2} d s\right]^{1 / 2}
\end{aligned}
$$

for any given $S>0, T>0$. Fix $T>0$ arbitrarily and let $S=T$. If $\varepsilon>0$, and

$$
\sup _{0 \leqq t \leqq T}\left|x(t)-x_{0}(t)\right|<\varepsilon /\left[T \sup _{0 \leqq t \leqq T}\left(\int_{0}^{t}\left|k(t, s) m_{T T}(s)\right|^{2} d s\right)^{1 / 2}\right]
$$

$\left|F x(t)-F x_{0}(t)\right|<\varepsilon$. We have used, again, Lemma 2.1.

THEOREM 2.4. Let the notation and hypotheses of Theorems 2.2 and 2.3 hold. Then there exists one and only one solution $x^{*} \in C\left(R^{+}\right)$of the equation (0.1). Further, $x^{*}$ is the limit in $C\left(R^{+}\right)$of the sequence of successive approximations $x_{n+1}=F x_{n}$, $x_{0}=\theta$.

Proof. Condition (2.9) implies $f(t, 0)=\theta$. Hence $F \theta=y$. Choose $x_{3} \in C\left(R^{+}\right)$such that $0 \leqq|y(t)| \leqq x_{3}(t), t \in R^{+}$. Let $H$ be the operator defined in Theorem 2.2, and define $H_{1} x=H(|x|)$. It is easy to see that

(i) $H_{1} x \geqq \theta$ and $H_{1}:[-x, x] \rightarrow\left[\theta, H_{1} x\right] \forall x \in C\left(R^{+}\right)$.

(ii) $|F x-F \theta| \leqq H_{1} x$.

(iii) The equation $x_{3}+H x=x$ has a solution $x_{1} \in C\left(R^{+}\right)$with $\theta \leqq x_{1}$ and hence $x_{3}+H x_{1}=x_{1}$.

By Theorem 1.2, $F:\left[-x_{1}, x_{1}\right] \rightarrow\left[-x_{1}, x_{1}\right]$. Now, for each $n \geqq 1$ set $S(n)$ $=\sup _{0 \leqq t \leqq n}\left|x_{1}(t)\right|$. Consider the sequence of functions $m_{S(n) n}$ determined by the hypothesis stated in Theorem 2.3 with $S=S(n), T=n$. Define

$$
m(t)=m_{S(n) n}(t) \text { for } n-1 \leqq t<n, n=1,2,3, \ldots
$$

By the properties of the functions $m_{S(n) n}$, it is immediate that $m(t)$ is locally $L^{2}$ compatible with $k$. Moreover, if $x^{\prime}, x^{\prime \prime} \in\left[-x_{1}, x_{1}\right]$, then

$$
\left|f\left(t, x^{\prime}(t)\right)-f\left(t, x^{\prime \prime}(t)\right)\right| \leqq m(t)\left|x^{\prime}(t)-x^{\prime \prime}(t)\right| \quad \forall t \in R^{+} .
$$

Let $\hat{k}(t, s)=(1 / \delta) k(t, s)$ for some $\delta, 0<\delta<1$. Then, applying Theorem 2.1, with $g=m$ and $k=\hat{k}$, we see that there is a unique $x_{2} \in C\left(R^{+}\right)$with $x_{2} \geqq 0$ satisfying

$$
x_{1}(t)+\frac{1}{\delta} \int_{0}^{t}|k(t, s) m(s)|\left|x_{2}(s)\right| d s=x_{2}(s) .
$$

Let $G: C\left(R^{+}\right) \rightarrow C\left(R^{+}\right)$be the operator defined by

$$
G x(t)=\int_{0}^{t}|k(t, s) m(s)||x(s)| d s .
$$


Conditions (a), (b), (c) (with $m=2$ ), and (e) of Theorem 1.1 hold for $G, x_{1}, x_{2}$ and $x_{3}$ found above. That condition (d) holds follows from

$$
|F x(t)-F y(t)| \leqq \int_{0}^{t}|k(t, s) m(s)||x(s)-y(s)| d s=G(x-y)(t)
$$

for all $x, y$ in $\left[-x_{1}, x_{1}\right]$. An application of Theorem 1.1 yields a solution $x^{*} \in C\left(R^{+}\right)$ for (0.1) unique in $\left[-x_{1}, x_{1}\right]$. That it is unique in $C\left(R^{+}\right)$is seen by observing that if $x^{* *}$ were a second solution, then $x_{3}$ could be chosen initially so that $x_{3} \geqq\left|x^{* *}\right| \geqq \theta$. In this case, then, $x^{* *}$ would lie in $\left[-x_{1}, x_{1}\right]$ as determined above, which then would result in a contradiction.

3. In the example of this section, we will not require that $f$ satisfy a Lipschitz condition, but rather one similar to one considered by Nohel [8]. We will need a lemma.

LEMMA 3.1. Let $h(t) \geqq 1, \varphi(t) \geqq 1$ be continuously differentiable on $R^{+}$with $h^{\prime}(t) \geqq 0$. Let $\psi$ be continuous, monotone nondecreasing on $R^{+}$, and for some $K>0$, assume $\psi(x)>0$ for $x \geqq K$. Then, if $\psi(x) \geqq x$ and

$$
\int_{K}^{\infty} \frac{1}{\psi(x)} d x=+\infty
$$

there exists $x_{1} \in C\left(R^{+}\right)$such that

$$
h(t)+h(t) \int_{0}^{t} \varphi(s) \psi\left(x_{1}(s)\right) d s \leqq x_{1}(t) .
$$

Proof. We define $x_{1}$ through the equation

$$
L(x, t)=\int_{H}^{x} \frac{1}{\psi(s)} d s-\int_{0}^{t}\left[1+\frac{h^{\prime}(s)}{h(s)}\right] \varphi(s) h(s) d s=0,
$$

where $H \geqq \max \{h(0), K\}$. That a unique solution $x=x_{1}(t)$ is defined for $t \geqq 0$ is clear from (3.3) and the fact that $\psi(x)>0, x \geqq H$. Since $\partial L / \partial x=1 / \psi(x)>0$, an application of the local Implicit Function Theorem to the equation $L(x, t)=0$ at $\left(x_{1}(t), t\right)$ yields a unique, continuously differentiable solution in a neighborhood of $t$. Since it must coincide with $x_{1}, x_{1}$ is a continuously differentiable function. Differentiating (3.3),

$$
x^{\prime}(t) / \psi\left(x_{1}(t)\right)=\left[1+h^{\prime}(t) / h(t)\right] h(t) \varphi(t) .
$$

Let $u(t)=x_{1}(t) / h(t)$. It is easy to show $u^{\prime}(t) \geqq u(t)$. Indeed,

$$
\begin{aligned}
u^{\prime}(t) & =(h(t))^{-2}\left[x_{1}^{\prime}(t) h(t)-h^{\prime}(t) x_{1}(t)\right] \\
& =(h(t))^{-2}\left[\psi\left(x_{1}(t)\right)\left(1+h^{\prime}(t) / h(t)\right)(h(t))^{2} \varphi(t)-h^{\prime}(t) x_{1}(t)\right] \\
& \geqq \psi(h(t) u(t))+[\psi(h(t) u(t))-u(t)]\left[h^{\prime}(t) / h(t)\right],
\end{aligned}
$$


using the fact that $\varphi(t) \geqq 1$ for the last inequality. Since $\psi$ is monotone and $\psi(x) \geqq x$, and $h(t) \geqq 1$ while $h^{\prime}(t) \geqq 0$, the last expression leads to

$$
u^{\prime}(t) \geqq h(t) u(t)+[h(t) u(t)-u(t)] h^{\prime}(t) / h(t) \geqq h(t) u(t) \geqq u(t) .
$$

We can now write

$$
\begin{aligned}
u^{\prime}(t)\left[\frac{h^{\prime}(t)}{h(t)}+1\right] & =\frac{u^{\prime}(t) h^{\prime}(t)+h(t) u^{\prime}(t)}{h(t)} \geqq \frac{u(t) h^{\prime}(t)+h(t) u^{\prime}(t)}{h(t)} \\
& \geqq \frac{[u(t) h(t)]^{\prime}}{h(t)}=\frac{x_{1}^{\prime}(t)}{h(t)}=\frac{\left[1+h^{\prime}(t) / h(t)\right] \psi\left(x_{1}(t)\right) h(t) \varphi(t)}{h(t)} .
\end{aligned}
$$

Dividing, we have $u^{\prime}(t) \geqq \psi\left(x_{1}(t)\right) \varphi(t)$. Integrating, $u(t) \geqq u(0)+\int_{0}^{t} \psi\left(x_{1}(s)\right) \varphi(s) d s$. Recalling the definition of $u$,

$$
\begin{aligned}
x_{1}(t) & =h(t) \frac{x_{1}(0)}{h(0)}+h(t) \int_{0}^{t} \psi\left(x_{1}(s)\right) \varphi(s) d s \\
& \geqq h(t)+h(t) \int_{0}^{t} \psi\left(x_{1}(s)\right) \varphi(s) d s .
\end{aligned}
$$

THEOREM 3.1. Let $h, \varphi, \psi$ and $x_{1}$ be as in the statement of Lemma 3.1. Suppose $f: R^{+} \times R \rightarrow R, k: R^{+} \times R^{+} \rightarrow R$ and $\psi$ satisfy

(3.5) (a) $\exists \delta, 0<\delta<1$, with $|k(t, s)| \leqq \delta h(t), s \geqq 0$,

(3.6) (b) $|f(t, x)-f(t, y)| \leqq \varphi(t) \psi(|x-y|)$,

(3.7) (c) $\exists m, 0<m \leqq 2$ such that $\psi(\lambda t) \leqq \lambda \psi(t)$ if $0<\lambda \leqq m$,

for $t \geqq 0$ and all $x, y$ in $R$. If $k$ is measurable, $f$ continuous, and $f(t, 0) \equiv 0$, then $(0.1)$ has a unique solution $x^{*} \in C\left(R^{+}\right)$, (in fact $\left.x^{*} \in\left[-(m / 2) x_{1},(m / 2) x_{1}\right]\right)$, for each $y \in C\left(R^{+}\right)$satisfying $|y(t)| \leqq m h(t) / 2, t \in R^{+}$. Moreover the solution is the limit in $C\left(R^{+}\right)$of the sequence of successive approximations $x_{0}=\theta, x_{n}=F\left(x_{n-1}\right), n \geqq 1, F$ defined by (2.10).

Proof. That, under the conditions of the theorem, $F: C\left(R^{+}\right) \rightarrow C\left(R^{+}\right)$continuously is easy to verify. Let $\hat{x}_{1}=(m / 2) x_{1} . F:\left[-\hat{x}_{1}, \hat{x}_{1}\right] \rightarrow\left[-\hat{x}_{1}, \hat{x}_{1}\right]$. For, if $G: C\left(R^{+}\right) \rightarrow C\left(R^{+}\right)$is defined by

$$
G x(t)=\int_{0}^{t}|k(t, s) \varphi(s)| \psi(|x(s)|) d s,
$$

then $G$ satisfies (a) and (b) of Theorem 1.1 and, clearly, $|F x-F \theta| \leqq G x$. Moreover, letting $x_{3}(t)=m h(t) / 2$,

$$
x_{3}+G\left(m x_{1} / 2\right) \leqq m x_{1} / 2
$$

by Lemma 3.1, noting that (c) of Theorem 1.1 holds by the homogeneity restriction on $\psi$. By Theorem 1.2, we see $F:\left[-(m / 2) x_{1},(m / 2) x_{1}\right] \rightarrow\left[-(m / 2) x_{1},(m / 2) x_{1}\right]$. Let $x_{2}=x_{1} \geqq(2 / m) \hat{x}_{1}$. Then, we see easily, $G x_{2}=G x_{1} \leqq \delta x_{1}=\delta x_{2}$. Therefore, $(1 / \delta) G x_{2}$ $\geqq x_{2}$. Since (d) of Theorem 1.1 follows easily from (3.6), an application of that theorem yields a solution $x^{*} \in C\left(R^{+}\right)$, unique in $\left[-(m / 2) x_{1},(m / 2) x_{1}\right]$. That $x^{*}$ is 
unique in $C\left(R^{+}\right)$can be seen as follows. Suppose $x^{* *}$ is a second solution in $C\left(R^{+}\right)$. In this case, we could choose $(m / 2) h(t) \geqq \max \left\{(1 / \delta)|k(t, s)|,|y(t)|,\left|x^{* *}(t)\right|\right\}$. Since, then, (3.8) implies $(m / 2) x_{1}(t) \geqq x_{3}(t)=(m / 2) h(t) \geqq\left|x^{* *}(t)\right|$, we have a contradiction to the uniqueness of the solution $x^{*}$ in $\left[-(m / 2) x_{1},(m / 2) x_{1}\right]$ obtained by successive approximations.

REMARK 3.1. While the condition (3.7) is a little unusual, it does include functions of the form $\psi(x)=x \varphi(x)$ where $\varphi$ is monotone increasing on $[0, \infty)$ with $m=1$. For example $\psi(x)=x \ln (1+x)+x$ satisfies all the conditions of the theorem. Notice also that $f(t, x)$ need not, then, be $O(|x|)$ as was assumed in $\S 2$.

REMARK 3.2. If $h(t)$ is bounded by a constant, say $H$, the proof of Lemma 3.1 can be simplified considerably. In fact, if $\hat{x}_{1}(t)$ is defined by

$$
\int_{H}^{\hat{x}_{1}(t)} \frac{1}{\psi(s)} d s=H \int_{0}^{t} \varphi(s) d s,
$$

then $x_{1}(t)=\left[\hat{x}_{1}(t) h(t)\right] / H$ satisfies the conclusion of Lemma 3.1. Notice that if $\varphi$ is integrable on $[0, \infty)$, then $\hat{x}_{1}$ and, hence, $x_{1}$ are bounded. This will result, then, through Theorem 3.1, in the assertion that there is a unique solution of $(0.1)$ in $C\left(R^{+}\right)$, and that solution is bounded.

\section{REFERENCES}

1. F. Brauer, Global behavior of solutions of ordinary differential equations, J. Math. Anal. Appl. 2 (1961), 145-158. MR 24 \#A284.

2. C. Corduneanu, Problèmes globaux dans la théorie des équations intégrales de Volterra, Ann. Mat. Pura Appl. (4) 67 (1965), 349-363. MR 32 \#331.

3. - Some perturbation problems in the theory of integral equations, Math. Systems Theory 1 (1967), 143-155. MR 35 \#4773.

4. V. Lakshmikantham and S. Leela, Differential and integral inequalities. Vol. 1, Academic Press, New York and London, 1969.

5. T. McDermott, Nonlinear mappings in locally convex spaces, Trans. Amer. Math. Soc. 153 (1971), 157-165.

6. - Implicitly defined mappings in locally convex spaces, Trans. Amer. Math. Soc. 161 (1971), 89-99.

7. R. K. Miller and G. R. Sell, Existence, uniqueness and continuity of solutions of integral equations, Ann. Mat. Pura Appl. (4) 80 (1968), 135-152. MR 40 \#663.

8. J. A. Nohel, Some problems in nonlinear Volterra integral equations, Bull. Amer. Math. Soc. 68 (1962), 323-329. MR 26 \#2838.

9. F. Smithies, Integral equations, Cambridge Tracts in Math. and Math. Phys., no. 49, Cambridge Univ. Press, New York, 1958. MR 21 \#3738.

10. A. Strauss, On a perturbed Volterra integral equation, J. Math. Anal. Appl. 30 (1970), 564-575. MR 41 \#5906.

Department of Mathematics, University of Hawail, Honolulu, Hawail 96822 